\title{
Propofol attenuates sepsis-induced acute kidney injury by regulating miR-290-5p/CCL-2 signaling pathway
}

\author{
Guodong Zheng ${ }^{1}$, Hong $\mathrm{Qu}^{2}$, Fen $\mathrm{Li}^{1}$, Weiquan $\mathrm{Ma}^{1}$ and Hong Yang ${ }^{1}$ \\ ${ }^{1}$ Department of Critical Care Medicine, Third Affiliated Hospital of Southern Medical University, Guangzhou, China \\ ${ }^{2}$ Department of Hematology, Guangzhou Panyu Central Hospital, Guangzhou, China
}

\begin{abstract}
Previous studies have indicated that propofol has immunomodulatory and antioxidative properties. However, the renoprotection effect and the precise mechanisms of propofol in sepsis-induced renal injury remain unclear. The purpose of the present study was to investigate the role of miR-290-5p/CCL-2 signaling in septic mice treatment with propofol. Mice were treated with propofol $(50 \mathrm{mg} / \mathrm{kg})$ twice within $24 \mathrm{~h}$. Survival outcome was monitored within $48 \mathrm{~h}$. The mRNA and protein levels were assayed by qRT-PCR and western blotting, respectively. Mouse podocytes (MPC5) were treated with lipopolysaccharide (LPS) to establish the cell model in vitro. The proliferation of MPC5 was monitored using the MTS assay. Cell apoptosis was analyzed by flow cytometry. Propofol improved survival outcome and alleviated acute kidney injury in cecal ligation and puncture-operated mice. Propofol increased miR-290-5p expression and decreased CCL-2 and inflammatory cytokines levels in the kidney for septic mice. We found that miR-290-5p was a direct regulator of CCL-2 in MPC5. Propofol could abrogate LPS-induced growth inhibition and apoptosis in MPC5. Meanwhile, propofol inhibited CCL-2 expression in LPS-treated MPC5, however, knockdown of miR-290-5p abrogated the inhibitory effect propofol on the mRNA and protein expressions of CCL-2. Propofol could serve as an effective therapeutic medication to suppress sepsis-induced renal injury in vivo and in vitro by regulating the miR-290-5p/ CCL-2 signaling pathway.
\end{abstract}

Key words: Propofol; Sepsis; miR-290-5p; CCL-2; Acute kidney injury

\section{Introduction}

Sepsis is a variety of serious infectious diseases with pathogens that can release bacterial toxin into the body and induce an inflammatory response in the host, leading to septic shock and multiple organ failure (1). Acute kidney injury $(\mathrm{AKI})$ is associated with approximately $70 \%$ mortality triggered by sepsis in the intensive care unit (ICU) (2). In addition, sepsis-associated AKI is associated strongly with a poor prognosis and poses significant clinical challenges for clinicians (3). To our knowledge, there is no effective therapeutic medication to improve the clinical outcomes of sepsis-associated AKI (4). Therefore, we need to explore novel drugs to protect the kidney from sepsis. Propofol has long been recognized as a rapid, short-acting intravenous anesthetic, widely used in clinical anesthesia as well as for sedation in the ICU (5). Recently, propofol has been reported to improve oxidative stress and inflammatory response in various tissues and organs, including lung, brain, and liver (6-9). In addition, propofol shows renoprotective effects in endotoxemia (10), ischemia-reperfusion (11), and orthotopic liver transplantation-induced AKI (12). In septic animal models, propofol treatment can protect the kidney from sepsis-induced AKI by decreasing inflammatory cytokines and inhibiting oxidative stress (13). However, potential molecular mechanisms of propofol in cecal ligation and puncture (CLP)-induced AKI have not been clearly delineated. A class of small non-coding RNAs (18-25 nucleotides), known as microRNAs (miR), have emerged as the post-translational modulators that regulate the translation of target messenger RNAs (mRNAs) by binding to its $3^{\prime}$-untranslated regions (3'-UTRs) (14). miRs are involved in a wide variety of diseases, including sepsis-associated AKI (15). Gene ontology (GO) analysis indicates that differentially expressed miRs in sepsisinduced $\mathrm{AKI}$ are primarily related to regulation of oxidative stress and mitochondrial dysfunction pathways (16). miRs also modulate the inflammatory response to endotoxin in mice (17). For example, miR27a is up-regulated and promotes inflammatory response in sepsis (18). miR-205-5b shows an anti-inflammatory activity in lipopolysaccharide

Correspondence: Hong Yang: <yanghong_1103@outlook.com> 
(LPS)-induced sepsis (19). In the present study, we aimed to determine the anti-inflammatory activity of propofol associated with miR-mediated post-transcriptional regulatory mechanism.

\section{Material and Methods}

\section{Animal treatment}

A total of 48 male 8-week-old C57BL/6J mice (body weight $20 \pm 2 \mathrm{~g}$ ) were obtained from the Third Affiliated Hospital of Southern Medical University (China) and allowed to acclimate to the environment for 1 week. The mice were given free access to food and tap water and were individually caged under controlled temperature $\left(23 \pm 2^{\circ} \mathrm{C}\right)$ and humidity $(55 \pm 5 \%)$ with an artificial $12-\mathrm{h}$ light/dark cycle. Sepsis in mice was induced by CLP surgical operation, as described previously (13). To investigate the effect of propofol during sepsis-induced AKI, the mice were randomly divided into 4 groups as follows: i) shamoperated mice $(n=12)$ as control group, ii) CLP group $(n=12)$ injected with normal saline, iii) propofol group $(n=12)$ that received propofol injection $(50 \mathrm{mg} / \mathrm{kg}$; twice within $24 \mathrm{~h}$; Sigma-Aldrich, USA) in sham-operated mice, and iv) propofol + CLP group that received propofol injection (50 mg/kg; twice within $24 \mathrm{~h}$ ) in CLP-operated mice $(n=12)$. Twenty-four hours after CLP surgical operation, mice were sacrificed by an overdose of sodium pentobarbital (2\%; $200 \mathrm{mg} / \mathrm{kg}$; Sigma-Aldrich). The blood from the hearts was collected for serum biochemical analysis. Kidneys were collected and immediately frozen in liquid nitrogen and $4 \%$ formalin at room temperature for gene and protein analysis and paraffin-embedded histological analysis, respectively. In another experiment, we observed the 48-h survival of CLP mice with or without propofol treatment ( $n=12$ in each group). This experiment was approved by the Ethics Committee of the Third Affiliated Hospital of Southern Medical University.

\section{Enzyme-linked immunosorbent assay (ELISA)}

Serum CCL-2 was measured by a mouse ELISA kit (Cat. No. E-EL-M0006c; Elabscience Biotechnology Co., Ltd, China). Glutamic oxaloacetic transaminase (GOT) (Cat. No. c010-1) and glutamic pyruvic transaminase (GPT) (Cat. No. c009-1) were determined using assay kits (Nanjing Jiancheng Biology Engineering Institute, China). In brief, $5 \mu \mathrm{L}$ serum and $25 \mu \mathrm{L}$ GOT or GPT substrates were added to 96 -well plates at $37^{\circ} \mathrm{C}$ for $30 \mathrm{~min}$. Then, 2,4-dinitrophenylhydrazine $(25 \mu \mathrm{L})$ was added to all wells at $37^{\circ} \mathrm{C}$ for $30 \mathrm{~min}$. Finally, sodium hydroxide $(0.4 \mathrm{~mol} / \mathrm{L} ; 250 \mu \mathrm{L})$ was added to stop the reactions at room temperature for $15 \mathrm{~min}$, and the absorbance was measured at $510 \mathrm{~nm}$. Serum creatinine (Cre; Cat. No. C011-1; Nanjing Jiancheng Biology Engineering Institute, China) and blood urea nitrogen (BUN; Cat. No. C013-2; Nanjing Jiancheng Biology Engineering Institute) levels were measured using an autoanalyzer and an enzymatic kinetic method using commercial kits following the manufacturer's protocol with a SpectraMax M5 ELISA plate reader (Molecular Devices, LLC, USA).

\section{Hematoxylin \& eosin (H\&E) staining}

Kidneys were collected and fixed with $4 \%$ formalin at room temperature for $24 \mathrm{~h}$, and paraffin-embedded. Tissues were cut into $3-\mu \mathrm{m}$ thick sections, which were stained with H\&E (Beyotime Institute of Biotechnology, China) at room temperature and visualized under a microscope (Leica DM 2500; Leica Microsystems GmbH, Germany). Renal injury was assessed using a previously described $0-4$ scale (20), in which 0 is none; $1,<10 \% ; 2$, $10-25 \%$; 3, 26-75\%; or 4 , $>75 \%$.

\section{RNA analysis and RT-qPCR}

Total RNA was extracted by TRIzol (Invitrogen, USA) according to the manufacturer's protocol. cDNA was synthesized by reverse transcription reactions with $2 \mu \mathrm{g}$ of total RNA using Moloney murine leukemia virus reverse transcriptase (Invitrogen; Thermo Fisher Scientific, USA) according to the manufacturer's protocol. PCR reaction mixtures $(20 \mu \mathrm{L})$ were prepared using the TaqMan Universal PCR Master Mix (Thermo Fisher Scientific) and performed using a DNA Engine (ABI 7300; Thermo Fisher Scientific). The reaction conditions were set according to the manufacturer's protocol. The $\mathrm{Cq}$ (quantification cycle fluorescence value) was calculated using SDS software, version 2.1 (Applied Biosystems; Thermo Fisher Scientific), and the relative expression levels of $\mathrm{miR}$ and $\mathrm{mRNA}$ were calculated using the $2^{-\Delta \Delta C t}$ method (21) and normalized to the internal control U6 and glyceraldehyde 3-phosphate dehydrogenase (GAPDH), respectively. The primers were synthesized by Sangon Biotech (China) as shown in Table 1. The PCR products were confirmed by $2 \%$ agarose (Sigma-Aldrich) gel electrophoresis and visualized under a gel imaging analysis system (Bio-Rad Laboratories, Inc., USA).

\section{Cell culture}

Mouse podocytes (MPC5) were obtained from the National Infrastructure of Cell Line Resource (Serial number: 3111C0001CCC000230; China) and maintained in RPMI1640 (Invitrogen, USA) supplemented with 10\% FBS (Invitrogen) at $37^{\circ} \mathrm{C}$ in a humidified incubator (Thermo Fisher Scientific), under $5 \% \mathrm{CO}_{2}, 95 \%$ air atmosphere. MPC5 were treated with lipopolysaccharide (LPS; $100 \mathrm{ng} / \mathrm{mL}$, Sigma-Aldrich), or combined with propofol $(100 \mu \mathrm{M})$ or miR$290-5 p$ inhibitors $(100 \mathrm{nM})$. All of the experiments were performed in triplicate.

\section{Transfection with miR-290-5p mimics and inhibitors}

The sequences of the miR-290-5p mimics (5'-ACU CAAACUAUGGGGGCACUUU-3') and anti-miR-290-5p (antisense inhibitor of miR-290-5p: 5'-AAAGUGCCCC CAUAGUUUGAGU-3') were synthesized by RiboBio (China). The MPC5 were transfected using Lipofectamine 
Table 1. Primers for RT-qPCR.

\begin{tabular}{lcc}
\hline Gene & \multicolumn{1}{c}{ Forward primer $\left(5^{\prime}-3^{\prime}\right)$} & Reverse primer $\left(5^{\prime}-3^{\prime}\right)$ \\
\hline miR-124 & TCGGCAGGTAAGGCACGCGGTG & TCAACTGGTGTCGTGGAGTCGGC \\
miR-290-5p & GCTGGGTTTCACGGGGGTATCAA & TCAACTGAGTGCCGTAGGGTGCG \\
miR-292-5p & AGCTGGGGTTTTCUCGGGGGUC & GACGTTGAGTCCGATGTACCCGTA \\
U6 & CTCGCTTCGGCAGCACATATACT & ACGCTTCACGAATTTGCGTGTC \\
CCL-2 & ATTTCCACACTTCTATGCCTCCT & ATCCAGTATGGTCCTGAAGATCA \\
IL-1 $\beta$ & ACCTTCCAGGATGAGGACATGA & CTAATGGGAACGTCACACACCA \\
TNF- $\alpha$ & TACTCCCAGGTTCTCTTCAAGG & GGAGGTTGACTTTCTCCTGGTA \\
NGAL & GATGTTGTTATCCTTGAGGCCC & CACTGACTACGACCAGTTTGCC \\
GAPDH & GCACCGTCAAGCTGAGAAC & TGGTGAAGACGCCAGTGGA \\
\hline
\end{tabular}

2000 (Invitrogen; Thermo Fisher Scientific) at a final concentration of $100 \mathrm{nM}$. At $48 \mathrm{~h}$ post-transfection, the cells were harvested for analysis.

\section{Luciferase reporter gene assay}

The potential binding sites between miR-290-5p and CCL-2 were obtained using online prediction software (miRanda-mirSVR; http://www.microrna.org/), miRDB (http:// www.mirdb.org/), and TargetScan (http://www.targetscan. org/). The wild-type (WT) and mutant-type (MUT) $3^{\prime}$-UTR of CCL-2 were synthesized by RiboBio (China) and inserted into the multiple cloning sites of the luciferase expressing pMIR-REPORT vector (Ambion; Thermo Fisher Scientific, Inc.). For the luciferase assay, MPC5 $\left(1 \times 10^{5}\right)$ was seeded into 24-well plates and co-transfected with luciferase reporter vectors containing the WT and MUT of CCL-2-3'-UTR $(0.5 \mu \mathrm{g})$ and mimics and inhibitors of miR-290-5p (100 nM) using Lipofectamine 2000 (Invitrogen; Thermo Fisher Scientific). Luciferase activity was measured using the Dual Luciferase Reporter ${ }^{\circledR}$ Assay System (Cat. No. E1960; Promega, USA) on a Luminoskan ${ }^{\mathrm{TM}}$ Ascent Microplate Luminometer (Thermo Fisher Scientific).

\section{MTS assay}

The proliferation of MPC5 was monitored using the MTS assay kit (Promega Corporation, USA). Absorbance was measured at $492 \mathrm{~nm}$ using an ELISA reader (MD SpectraMax M5; Molecular Devices, LLC, USA).

\section{Flow cytometry analysis}

MPC5 was treated with LPS $(100 \mathrm{ng} / \mathrm{mL})$, LPS $(100 \mathrm{ng} / \mathrm{mL})+$ propofol $(100 \mu \mathrm{M})$, or LPS $(100 \mathrm{ng} / \mathrm{mL})+$ propofol $(100 \mu \mathrm{M})+$ miR-290-5p inhibitors $(100 \mathrm{nM})$ for $24 \mathrm{~h}$. Cells were collected after digestion and were washed twice with PBS and centrifuged at $1200 \mathrm{~g}$ for $5 \mathrm{~min}$ at $4^{\circ} \mathrm{C}$. The supernatant was discarded, and the cells were then resuspended and fixed in ice-cold $75 \%$ ethanol and stored at $4^{\circ} \mathrm{C}$. Annexin V-FITC apoptosis detection kit was purchased from Invitrogen. The samples were analyzed using flow cytometer (BD Biosciences, USA). The data were processed by Cell Quest Software (version 5.1, BD Biosciences,).

\section{Western blotting}

Proteins were extracted with radio immunoprecipitation assay (RIPA) buffer (Cat. No. P0013B; Beyotime Institute of Biotechnology), and the concentrations were determined using the Bicinchoninic Acid Kit for Protein Determination (Cat. No. BCA1-1KT; Sigma-Aldrich; Merck $\mathrm{KGaA})$. Protein $(30 \mu \mathrm{g})$ for each sample was separated on a $10 \%$ SDS-PAGE gel and transferred to nitrocellulose membranes (Bio-Rad Laboratories, Inc., USA). The membranes were incubated with the primary antibody CCL-2 (Cat. No. sc-1784; dilution: 1: 1,000; Santa Cruz Biotechnology, USA), and NF-kB/p65 (Cat. No. 3034; dilution: 1: 500; Cell Signaling Technology, Inc., USA). Following three washes with TBST, the membranes were incubated with the appropriate horseradish peroxidase-conjugated secondary antibody (Cat. No. sc-516102; dilution: 1:10,000; Santa Cruz Biotechnology) at room temperature for $2 \mathrm{~h}$ and visualized by chemiluminescence (Thermo Fisher Scientific, Inc.). Signals were analyzed with Quantity One ${ }^{\mathbb{R}}$ software version 4.5 (Bio Rad Laboratories, Inc.). GAPDH (Cat. no: 2118; dilution: 1: 2,000; Cell Signaling Technology, Inc., USA) and histone (Cat. no: 9715; dilution: 1: 2,000; Cell Signaling Technology, Inc.) were used as control antibodies.

\section{Statistical analysis}

Data are reported as the mean \pm SD for each group. All statistical analyses were performed using PRISM version 5.0 (GraphPad Software, Inc., USA). Statistical differences between two groups were determined using Student's $t$-test. Inter-group differences were analyzed by one-way analysis of variance, followed by a post-hoc Tukey's test for multiple comparisons. Survival rates were calculated using the Kaplan-Meier method with the logrank test applied for comparison. $\mathrm{P}<0.05$ was considered to indicate a statistically significant difference.

\section{Results}

\section{Propofol had a significant effect on sepsis-induced AKI in mice}

In our study, CLP surgical operation was performed to establish the polymicrobial sepsis-induced AKI in mice. 
Table 2. Effects of propofol on hepatic and renal function in cecal ligation and puncture (CLP)-operated or sham-operated mice.

\begin{tabular}{lcccc}
\hline & Sham & CLP & Propofol & Propofol + CLP \\
\hline CCL-2 $(\mathrm{pg} / \mathrm{mL})$ & $136.49 \pm 15.27$ & $321.67 \pm 48.34^{*}$ & $151.82 \pm 16.37$ & $198.61 \pm 31.47^{\#}$ \\
GOT $(\mathrm{U} / \mathrm{L})$ & $67.49 \pm 7.15$ & $432.67 \pm 78.93^{*}$ & $73.83 \pm 10.52$ & $173.56 \pm 25.73^{\#}$ \\
GPT $(\mathrm{U} / \mathrm{L})$ & $38.47 \pm 5.35$ & $205.71 \pm 36.72^{*}$ & $45.84 \pm 6.21$ & $75.49 \pm 13.51^{\#}$ \\
BUN $(\mathrm{mmol} / \mathrm{L})$ & $5.78 \pm 0.62$ & $17.27 \pm 2.31^{*}$ & $4.89 \pm 0.71$ & $9.12 \pm 1.68^{\#}$ \\
Cre $(\mu \mathrm{mol} / \mathrm{L})$ & $4.31 \pm 0.51$ & $54.65 \pm 10.95^{*}$ & $5.36 \pm 0.47$ & $20.61 \pm 4.22^{\#}$ \\
\hline
\end{tabular}

Data are reported as the means $\pm S D$. ${ }^{*} P<0.05$ compared with the Sham group; ${ }^{*} P<0.05$ compared with the CLP group (ANOVA). GOT: glutamic oxaloacetic transaminase; GPT: glutamic pyruvic transaminase; BUN: blood urea nitrogen; Cre: creatinine.

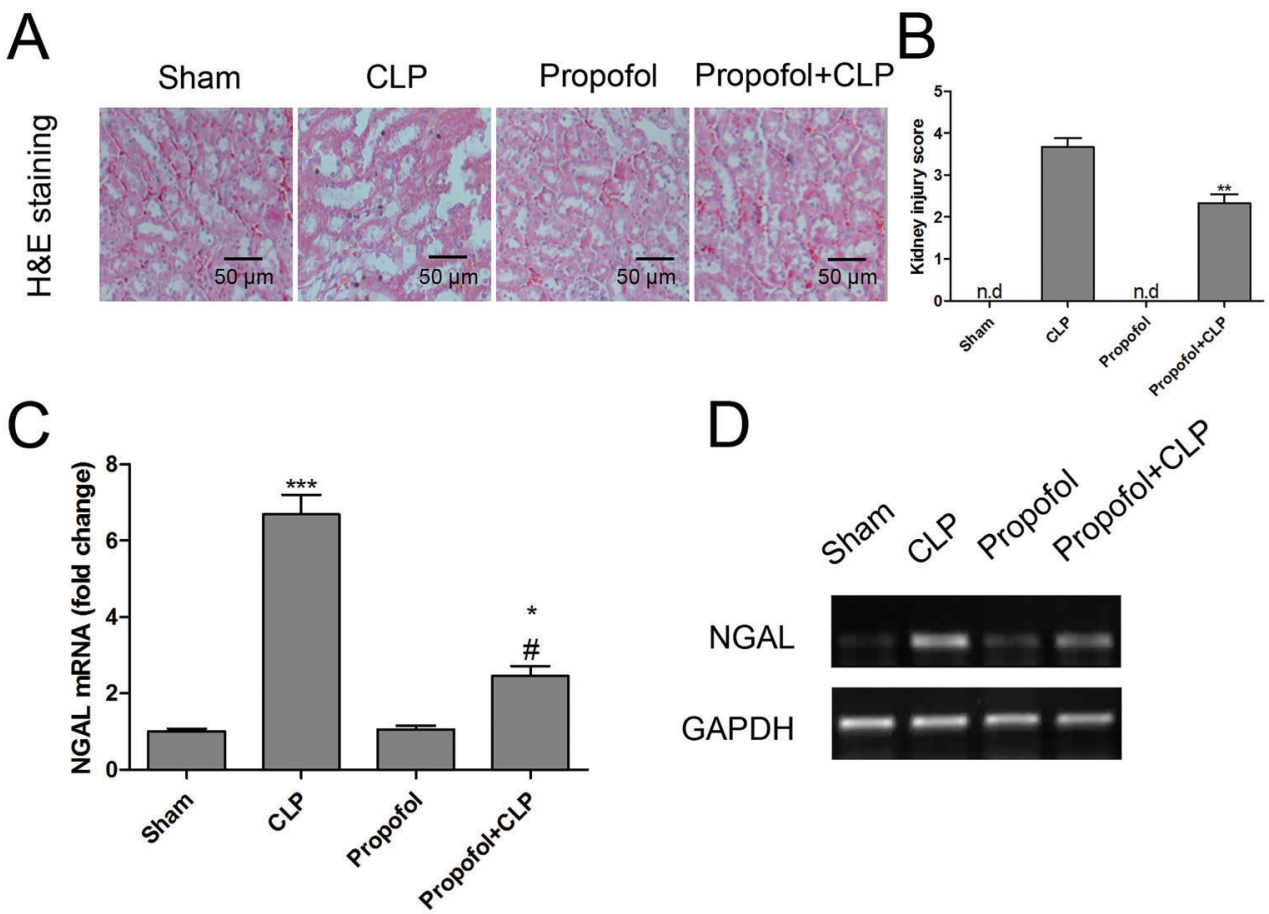

Figure 1. Propofol attenuated cecal ligation and puncture (CLP)-induced acute kidney injury in mice. Twenty-four hours after CLP surgical operation, renal histology (magnification $200 \times ; A$ ) and injury score $(B)$ were performed in the kidneys from mice with or without propofol treatment. Renal expression of neutrophil gelatinase associated lipocalin (NGAL) mRNA (relative to the controls) was measured by RT-qPCR $(C)$ and verified by agarose gel electrophoresis analysis $(D)$. Data are reported as means $\pm S D(n=12$ per group). ${ }^{*} \mathrm{P}<0.05,{ }^{* *} \mathrm{P}<0.01$, ${ }^{* * *} \mathrm{P}<0.001$ compared with the control group; ${ }^{\#} \mathrm{P}<0.05$ compared with the CLP group (ANOVA). nd: not detected.

Twenty-four hours after CLP surgical operation, the serum levels of GOT, GPT, BUN, and Cre were significantly higher in CLP-operated mice than in the control group, while propofol treatment decreased those levels in CLP-operated mice (Table 2). In addition, H\&E staining was performed to observe the extent of renal injury. As indicated in Figure $1 \mathrm{~A}$ and $\mathrm{B}$, the severe architectural disruptions of kidney were triggered by CLP surgical operation, including tubular dilatation and brush border loss. Renal injury scores were significantly increased in the CLP group compared with the control group. In contrast, propofol treatment preserved the morphologic integrity of kidney in CLP-operated mice. Neutrophil gelatinase associated lipocalin (NGAL) is a highly predictive biomarker of AKI (22). In the present study, the mRNA expression of NGAL was measured in the kidney from septic mice with or without propofol treatment. The results demonstrated that NGAL mRNA increased by 7 -fold after CLP surgical operation, but propofol treatment could reverse the mRNA expression of NGAL induced by CLP in the kidney from mice (Figure 1C). Furthermore, the PCR products of NGAL were confirmed by $2 \%$ agarose gel electrophoresis (Figure 1D). 


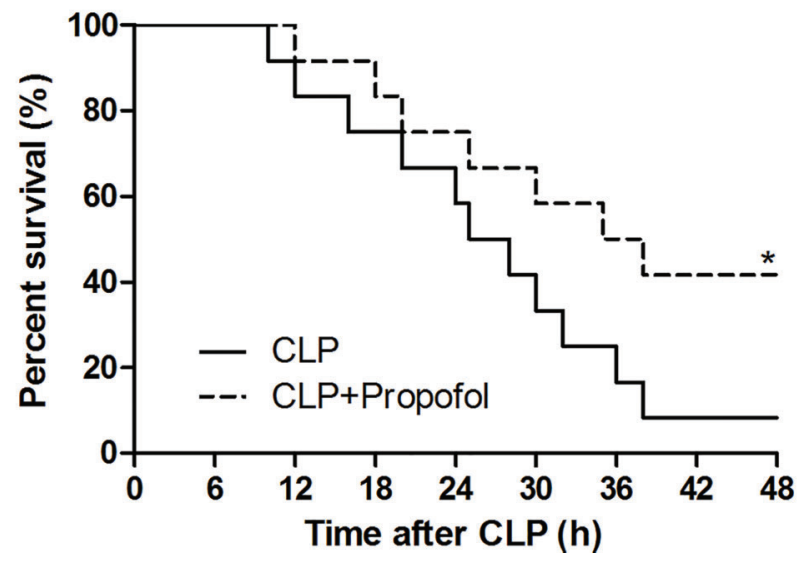

Figure 2. Propofol improved survival outcome in septic mice. Kaplan-Meier survival curves of cecal ligation and puncture (CLP)operated mice treated or non-treated with propofol. Data are reported as means $\pm S D\left(n=12\right.$ per group). ${ }^{*} P<0.05$ compared with the CLP group (log-rank test).

\section{Propofol improved survival outcome in CLP-operated mice}

We observed the 48-h survival of CLP mice with and without propofol treatment. We found that $92 \%$ of mortality occurred within $48 \mathrm{~h}$ after CLP surgical operation. However, the septic mice treated with propofol did not die at $38 \mathrm{~h}$, and $42 \%$ survived with propofol treatment (Figure 2 ).

\section{Propofol inhibited inflammatory gene expression in CLP-operated mice}

Induction of CCL-2 regulates some inflammatory cytokines levels, such as interleukin $1 \beta$ (IL-1 $\beta$ ), IL-6, and tumor necrosis factor $\alpha(T N F-\alpha)$ (23). In septic mice, CCL-2 induces systemic inflammatory response and promotes tissue repair (24). The plasma concentration of CCL-2 in sepsis patients is significantly higher than in healthy controls (25). In our study, we found that the serum concentration and mRNA of CCL-2 in CLP-operated mice were significantly up-regulated compared to shamoperated mice, while propofol treatment decreased the levels of CCL-2 in septic mice (Table 2, Figure $3 A$ and $B$ ). Moreover, septic mice exhibited significantly higher mRNA expression of IL-1 $\beta$ and TNF- $\alpha$ compared with the shamoperated mice. Propofol treatment also inhibited sepsisinduced inflammatory response (Figure $3 A, C$ and D). In addition, we detected the protein expression of NF-Kb (p65) in the nucleus and found an increased NF- $\mathrm{B}(\mathrm{p} 65)$ level in the kidney from CLP-operated mice, while propofol had the capacity to reduce CLP-induced up-regulation of $\mathrm{NF}-\mathrm{Kb}$ (p65) protein in the nucleus (Figure 3E). NF- $\mathrm{kB}$ as a key transcription factor has been implicated in the process of sepsis-induced inflammatory response. Over-activation of NF-KB is associated with cytoplasmic degradation of its inhibitor $I_{\kappa} \mathrm{B} \alpha$, which leads to the translocation of $\mathrm{p} 65$, a subunit of NF-kB, into the nucleus that binds to DNA and enhances the expression of inflammatory cytokines (26).

\section{Propofol was associated with post-transcriptional regulatory mechanism in septic mice}

Based on the above findings, we concluded that CCL-2 played a significant role in the pathogenesis of sepsis. To investigate whether CCL-2 could be regulated by miRs in sepsis-induced $\mathrm{AKI}$, the online prediction softwares miRanda-mirSVR, miRDB, and TargetScan were used for prediction. We found that miR-124, miR-290-5p, and miR-292-5p were overlapped in at least two databases. Therefore, we measured their expression in the kidney from septic mice with or without propofol treatment. The results demonstrated that NGAL miR-124 and miR-290-5p decreased by $47 \%$ and $78 \%$, respectively, after CLP surgical operation, which were restored by propofol treatment in septic mice. However, miR-292-5p had no obvious difference among the four groups (Figure 4). Therefore, we focused on miR-290-5p in our study.

First, the conserved binding sites between miR-290-5p and CCL-2 were depicted based on miRanda-mirSVR, miRDB, and TargetScan databases and are shown in Figure 5A. Then, the luciferase reporter plasmids containing WT or MUT $3^{\prime}-\mathrm{UTR}$ of CCL-2 were constructed. In addition, MPC5 were cotransfected with miR-290-5p mimics and luciferase reporter plasmid. Luciferase reporter assay showed that the luciferase activity of WT $3^{\prime}-U T R$ of CCL-2 reduced by nearly $56 \%$ with the co-transfection of miR-290-5p mimics. Transfected with miR-290-5p mimics, the luciferase enzyme activity had no significant change in the reporter vector containing MUT $3^{\prime}-$ UTR of CCL-2 (Figure 5B). We also found that the mRNA (Figure 5C) and protein (Figure 5D) expression of CCL-2 were markedly inhibited in MPC5 transfected with miR-290-5p mimics. As expected, the luciferase activity (Figure 5E), mRNA (Figure 5F), and protein (Figure 5G) levels of CCL-2 were dramatically increased after transfection with miR-290-5p inhibitors.

\section{Propofol improved LPS-induced MPC5 dysfunction by regulating miR-290-5p/CCL-2 signaling pathway}

To investigate the role of miR-290-5p in LPS and propofol-treated MPC5 podocytes, we found that transfection of miR-290-5p inhibitors significantly down-regulated the expression of miR-290-5p in MPC5 podocytes (Figure 6A). Potential cytotoxicity of LPS and propofol was analyzed using an MTS assay. The results indicated that propofol protected against LPS-induced MPC5 death, however, knockdown of miR-290-5p abrogated the protective effect of propofol on cell viability (Figure 6B). Intriguingly, similar results were obtained by flow cytometry analysis (Figure 6C and D). We also found that knockdown of miR-290-5p abrogated the inhibitory effect of propofol on the mRNA (Figure 6E) and protein (Figure 6F) expression of CCL-2. 

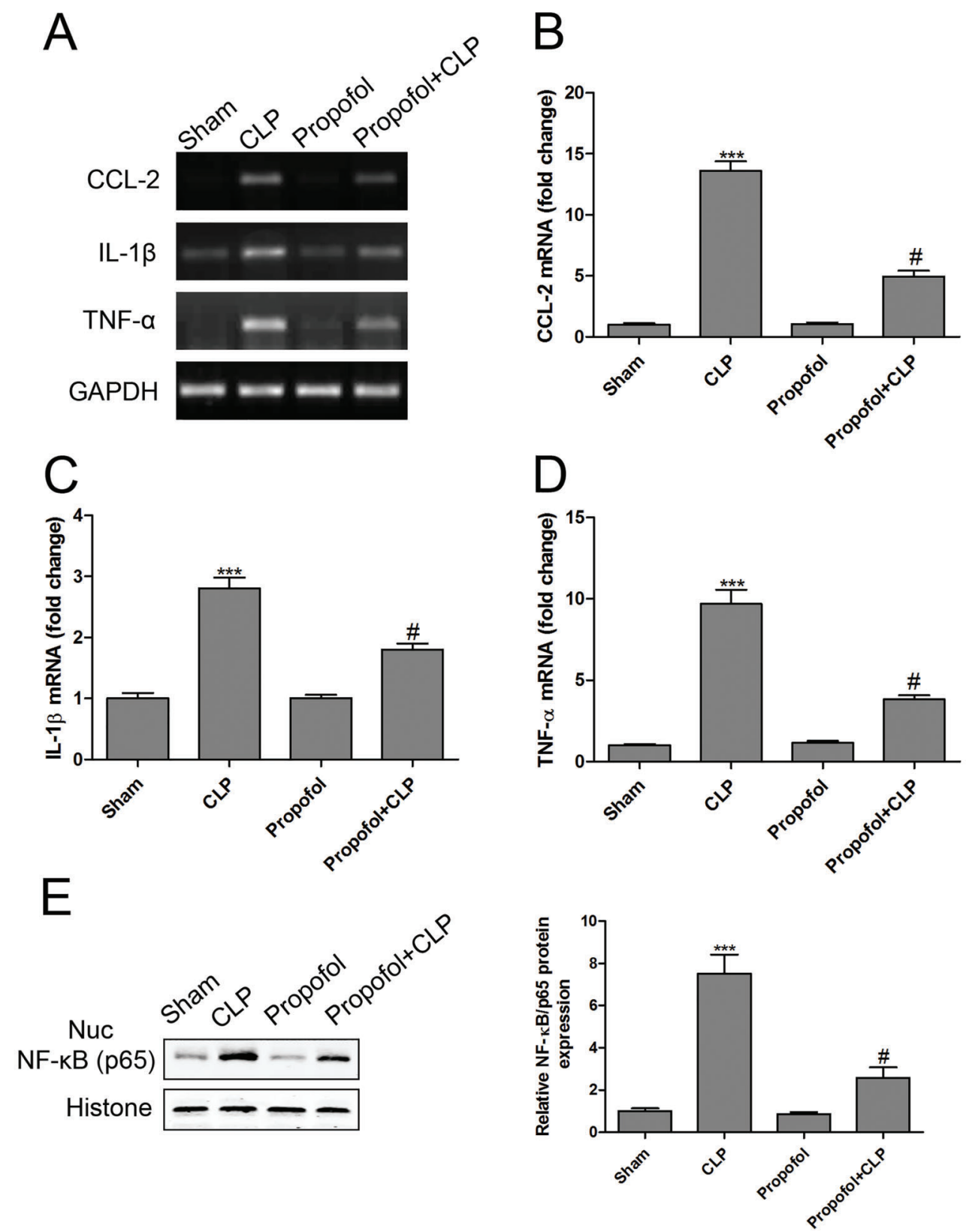

Figure 3. Propofol inhibited excessive inflammatory responses in septic mice. Renal mRNA levels of CCL-2 ( $A$ and $B$ ), interleukin (IL)-1 $\beta$ $(A$ and $C)$, and tumor necrosis factor $\alpha(\mathrm{TNF}-\alpha)(A$ and $D)$ were measured by RT-qPCR and verified by agarose gel electrophoresis analysis. Protein expression of NF-kB (p65) in the nucleus (Nuc) was measured by western blotting $(E)$. Data are reported as means $\pm S D$ ( $n=12$ per group). ${ }^{* \star} \mathrm{P}<0.001$ compared with the control group; ${ }^{\#} \mathrm{P}<0.05$ compared with the cecal ligation and puncture (CLP) group (ANOVA)

\section{Discussion}

It is well known that the inflammatory response plays a central role in the complications that develop in experimental sepsis (27). In septic mice with AKI, CLP leads to formation of oxidative stress, which appears to intensify inflammatory response (13). Suzuki et al. (28) showed that oxidative stress results in an increase in the expression of CCL-2, which accelerates macrophage recruitment, an inflammatory process. Similar mechanisms were seen in our study; we found that CLP induced the expression of CCL-2 and the subsequent increase of the production of proinflammatory cytokines, which might be associated with renal injury in septic mice. Importantly, 


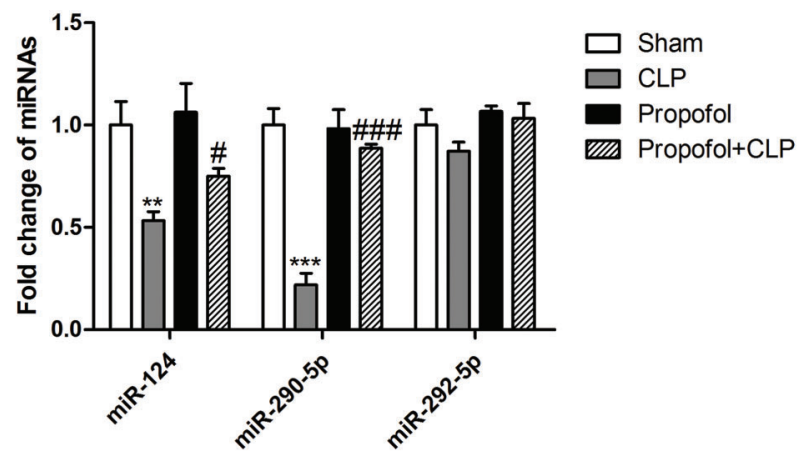

Figure 4. Effects of propofol $24 \mathrm{~h}$ after cecal ligation and puncture (CLP) on renal expression of miR-124, miR-290-5p, and miR-292-5p was measured by RT-qPCR in kidneys. Data are reported as means \pm SD ( $n=12$ per group). ${ }^{* \star} \mathrm{P}<0.01,{ }^{* \star *} \mathrm{P}<0.001$ compared with the sham group; ${ }^{\#} \mathrm{P}<0.05,{ }^{\# \#} \mathrm{P}<0.001$ compared with the CLP group (ANOVA). the post-transcriptional regulatory mechanism demonstrated that miR-290-5p could reversely regulate the expression of CCL-2. The in vivo model showed that propofol increased miR-290-5p levels as well as decreased the expression of CCL-2 in CLP-operated mice. The in vitro cell model confirmed that propofol protected LPS-induced MPC5 death by inhibiting CCL-2 levels. However, miR-290$5 p$ loss-of-function abrogated the protective effect of propofol on LPS-induced MPC5 apoptosis. All of these findings suggest that propofol can serve as an effective therapeutic medication to suppress sepsis-induced renal injury in vivo and in vitro by activating miR-290-5p and the subsequent inhibiting CCL-2 and its downstream pathways, such as the inflammatory response.

Propofol has been shown to be capable of antiinflammatory and anti-apoptotic effects, which may be attributed to its structural similarity to anti-inflammatory

A

miR-290-5p 3'-UUUCACGGGGGUAUCAAACUCA-5'

CCL-2 (WT) 5'-GGGUAAACUUUGU--GUUUGAGA-3'

CCL-2 (MUT) 5'-GGGUAAACUUUGU--GUAACUCA-3'

B

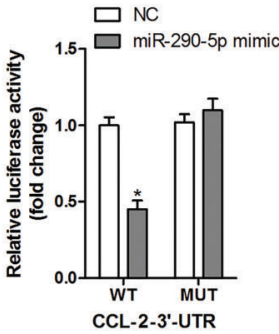

E

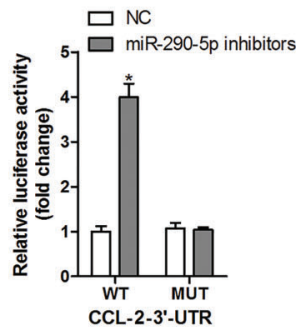

C

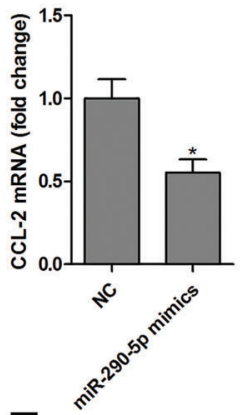

$\mathrm{F}$

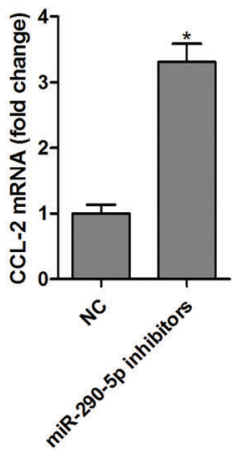

CCL-2

GAPDH
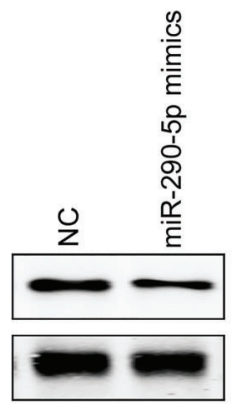

G

$\mathrm{CCL}-2$

GAPDH

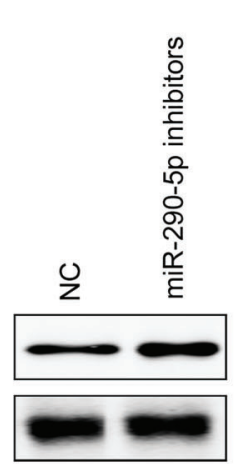

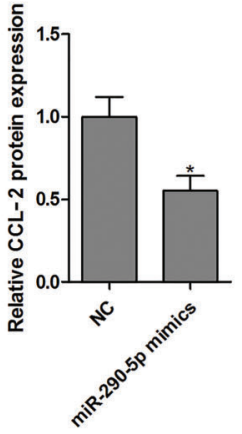

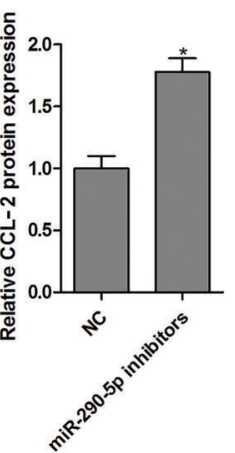

Figure 5. CCL-2 is a direct target gene of miR-290-5p. Schematic representation of the putative miR-290-5p binding site in the $3^{\prime}$-untranslated regions ( $3^{\prime}$ UTR) of CCL-2 was predicted by the online database $(A)$. Mouse podocytes cells (MPC5) were co-transfected with the wild type (WT) and mutant (MUT) of CCL-2-3'-UTR and miR-290-5p mimics, and luciferase activity $(B)$, mRNA $(C)$, and protein $(D)$ of CCL-2 were measured. After transfection with the WT and MUT of CCL-2-3'-UTR and miR-290-5p inhibitors, luciferase activity $(E)$, mRNA $(F)$, and protein $(G)$ of CCL-2 were measured. Data are reported as means $\pm S D$ ( $n=3$ per group). ${ }^{*} P<0.05$ compared with the normal control (NC) group (ANOVA). 

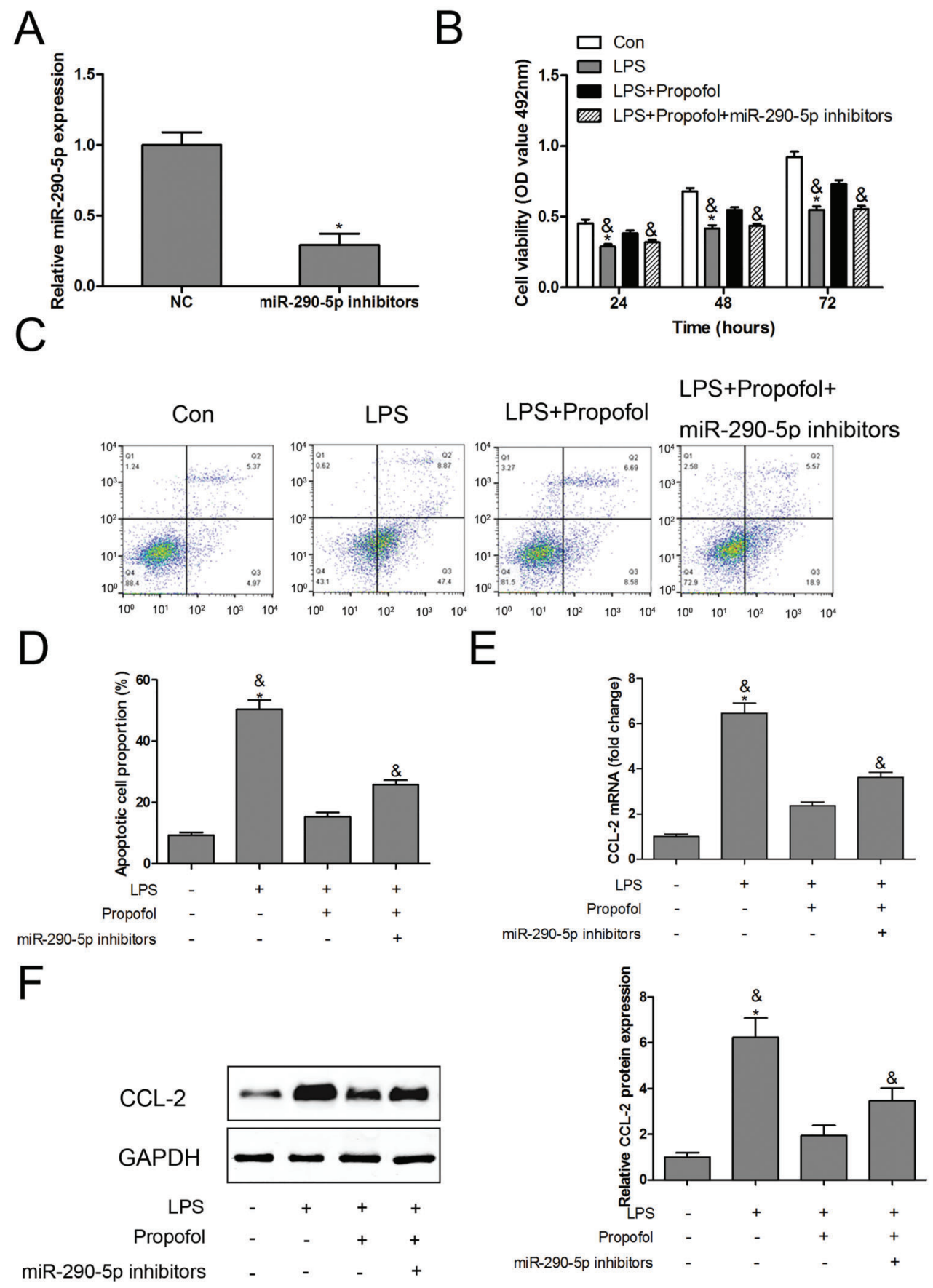

Figure 6. Inhibition of miR-290-5p neutralized the protective effect of propofol in lipopolysaccharide (LPS)-treated mouse podocytes (MPC5). The levels of miR-290-5p were measured by RT-qPCR after being transfected with miR-290-5p inhibitors in MPC5 (A). In LPStreated MPC5 combined with propofol or propofol + miR-290-5p inhibitors, cell viability was measured using the MTS assay $(B)$; apoptosis was analyzed using flow cytometry $(C$ and $D)$; the mRNA $(E)$ and protein $(F)$ expression of CCL-2 were detected by RT-qPCR and western blotting, respectively. Data are reported as the means $\pm S D$ ( $n=3$ in each group). $P<0.05$ compared with control group; ${ }^{\&} \mathrm{P}<0.05$ compared with LPS + propofol group. 
medications (10). Consistent with previous studies $(11,13)$, our results indicated that propofol treatment was shown to inhibit inflammatory reaction in vivo and attenuate apoptosis in vitro by targeting miR-290-5p/CCL-2 signaling pathway. Notably, CLP-treated mice or LPS-treated podocytes have increased expression of CCL-2, suggesting that CCL-2 may be central in the pathological process of renal injury, as reported previously $(13,29)$. We proposed the mechanism for the protective role of propofol, which protected against CLP or LPS-induced renal injury by inactivation of CCL-2 and its downstream inflammatory cytokines.

Further study on molecular mechanisms have examined the effects of propofol on miRs expression. In the present study, propofol significantly increased the expression of miR-290-5p in the kidney from septic mice. miR$290-5 p$ is a member of the miR-290-295 cluster, which are the most abundant miRs and mediate a latent pro-survival function in mouse embryonic stem cells (mESCs) $(30,31)$. miR-290-295 cluster deficiency results in partially penetrant embryonic lethality and germ cell defects in mice (32). In addition, miR-290-295 cluster has been found to accelerate cell proliferation by promoting the $\mathrm{G} 1$ to $\mathrm{S}$ phase transition (33), suggesting a role for this miR cluster in serving a protective function in preventing mESCs apoptosis. The anti-apoptotic functional consequence of miR290-5p in LPS-treated podocytes was confirmed by our in vitro experiments, which provided evidence that miR290-5p may decrease the renal injury by suppressing podocytes apoptosis. On the other hand, direct damage to renal tissues or cells by CLP or LPS might be alleviated through miR-290-5p-targeted CCL-2 and inflammatory cytokines. To our knowledge, this is the first study to report the anti-inflammatory activity of miR-290-5p in the animal and cell model of sepsis. Preliminary study has shown that improvement of survival rates and renal damage and

\section{References}

1. Hotchkiss RS, Monneret G and Payen D. Sepsis-induced immunosuppression: from cellular dysfunctions to immunotherapy. Nat Rev Immunol 2013; 13: 862-874, doi: 10.1038/ nri3552.

2. Schrier RW, Wang W. Acute renal failure and sepsis. N Engl J Med 2004; 351: 159-169, doi: 10.1056/NEJMra032401.

3. Bagshaw SM, Langenberg C, Wan L, May CN, Bellomo R. A systematic review of urinary findings in experimental septic acute renal failure. Crit Care Med 2007; 35: 15921598, doi: 10.1097/01.CCM.0000266684.17500.2F.

4. Alobaidi R, Basu RK, Goldstein SL, Bagshaw SM. Sepsisassociated acute kidney injury. Semin Nephrol 2015; 35: 2-11, doi: 10.1016/j.semnephrol.2015.01.002.

5. Borgeat A, Wilder-Smith $\mathrm{OH}$, Suter PM. The nonhypnotic therapeutic applications of propofol. Anesthesiology 1994; 80: 642-656, doi: 10.1097/00000542-199403000-00022

6. Gokcinar D, Ergin V, Cumaoglu A, Menevse A, Aricioglu A. Effects of ketamine, propofol, and ketofol on proinflammatory cytokines and markers of oxidative stress in a rat model inhibition of inflammation by propofol treatment in septic mice may be associated with up-regulation of miR-290-5p. These results suggest that miR-290-5p may be a potential synergist with propofol to increase survival rates in septic mice. A previous study found that miR-125b inserted into a lentivirus expression vector could prevent cardiac dysfunction and improve survival rates (approximately 30\%) in septic mice (34). Therefore, we hypothesized that lentivirusexpressed miR-290-5p may be a potential synergist with propofol. However, these conclusions need to be validated by in vivo experiments.

There are some limitations in our study. Although our results indicated that propofol inhibited CLP-induced AKI by enhancing miR-290-5p expression, a direct renoprotection of exogenous miR-290-5p in septic mouse model remains undefined. In addition, the regulating effect of CCL-2 on inflammatory cytokines might exist in the progression of sepsis-associated AKI, but the effect of CCL-2 gain-offunction or loss-of-function on inflammatory response is still not elucidated. Thus, the results obtained here only exhibit the possible therapeutic target of propofol in sepsis-induced AKI.

In conclusion, propofol decreased inflammatory cytokines in CLP-operated mice and inhibit apoptosis in LPStreated podocytes. In addition, propofol also protected the kidney from sepsis-induced injury, and the underlying mechanism was mediated, at least partially, by regulation of miR-290-5p/CCL-2 signaling pathway. These findings indicated that propofol might be used as a promising therapeutic agent for preventing sepsis-induced $\mathrm{AKI}$ in routine clinical practice.

\section{Acknowledgment}

This work was supported by the National Key Research and Development Program of China (2016YFC1201704). of endotoxemia-induced acute lung injury. Acta Biochim Pol 2013; 60: 451-456.

7. Romuk E, Szczurek W, Nowak P, Skowron M, Prudel B, Hudziec E, et al. Effects of propofol on oxidative stress parameters in selected parts of the brain in a rat model of Parkinson disease. Postepy Hig Med Dosw (online) 2016; 70: 1441-1450.

8. Ranjbar A, Sharifzadeh M, Karimi J, Tavilani H, Baeeri M, Heidary Shayesteh T, et al. Propofol attenuates toxic oxidative stress by ccl4 in liver mitochondria and blood in rat. Iran $J$ Pharm Res 2014; 13: 253-262.

9. Han D, Li S, Xiong Q, Zhou L, Luo A. Effect of propofol on the expression of MMP-9 and its relevant inflammatory factors in brain of rat with intracerebral hemorrhage. Cell Biochem Biophys 2015; 72: 675-679, doi: 10.1007/s12013-015-0516-9.

10. Cui WY, Tian AY, Bai T. Protective effects of propofol on endotoxemia-induced acute kidney injury in rats. Clin Exp Pharmacol Physiol 2011; 38: 747-754, doi: 10.1111/j.14401681.2011.05584.x. 
11. Li Y, Zhong D, Lei L, Jia Y, Zhou H, Yang B. Propofol prevents renal ischemia-reperfusion injury via inhibiting the oxidative stress pathways. Cell Physiol Biochem 2015; 37 : 14-26, doi: 10.1159/000430329.

12. Luo C, Yuan D, Li X, Yao W, Luo G, Chi X, et al. Propofol attenuated acute kidney injury after orthotopic liver transplantation via inhibiting gap junction composed of connexin 32 . Anesthesiology 2015; 122: 72-86, doi: 10.1097/ALN.000000 0000000448.

13. Hsing $\mathrm{CH}$, Chou W, Wang JJ, Chen $\mathrm{HW}$, Yeh $\mathrm{CH}$. Propofol increases bone morphogenetic protein-7 and decreases oxidative stress in sepsis-induced acute kidney injury. Nephrol Dial Transplant 2011; 26: 1162-1172, doi: 10.1093/ndt/gfq572.

14. O'Connell RM, Rao DS, Baltimore D. microRNA regulation of inflammatory responses. Annu Rev Immunol 2012; 30: 295-312, doi: 10.1146/annurev-immunol-020711-075013.

15. Li XY, Zhang K, Jiang ZY, Cai LH. MiR-204/miR-211 downregulation contributes to candidemia-induced kidney injuries via derepression of Hmx1 expression. Life Sci 2014; 102: 139-144, doi: 10.1016/j.Ifs.2014.03.010.

16. Ge QM, Huang CM, Zhu XY, Bian F, Pan SM. Differentially expressed miRNAs in sepsis-induced acute kidney injury target oxidative stress and mitochondrial dysfunction pathways. PLoS One 2017; 12: e0173292, doi: 10.1371/journal.pone.0173292.

17. Alexander M, Hu R, Runtsch MC, Kagele DA, Mosbruger $\mathrm{TL}$, Tolmachova $\mathrm{T}$, et al. Exosome-delivered microRNAs modulate the inflammatory response to endotoxin. Nat Commun 2015; 6: 7321, doi: 10.1038/ncomms8321.

18. Wang Z, Ruan Z, Mao $Y$, Dong $W$, Zhang $Y$, Yin $N$, et al. miR-27a is up regulated and promotes inflammatory response in sepsis. Cell Immunol 2014; 290: 190-195, doi: 10.1016/ j.cellimm.2014.06.006.

19. Zhou W, Wang J, Li Z, Li J, Sang M. MicroRNA-2055b inhibits HMGB1 expression in LPS-induced sepsis. Int $\mathrm{J} \mathrm{Mol}$ Med 2016; 38: 312-318, doi: 10.3892/ijmm.2016.2613.

20. Fan HY, Qi D, Yu C, Zhao F, Liu T, Zhang ZK, et al. Paeonol protects endotoxin-induced acute kidney injury: potential mechanism of inhibiting TLR4-NF-kappaB signal pathway. Oncotarget 2016; 7: 39497-39510, doi: 10.18632/onco target.8347

21. Livak KJ, Schmittgen TD. Analysis of relative gene expression data using real-time quantitative PCR and the 2(-Delta Delta C(T)) Method. Methods 2001; 25: 402-408, doi: 10.1006/ meth.2001.1262.

22. Han M, Li Y, Liu M, Li Y, Cong B. Renal neutrophil gelatinase associated lipocalin expression in lipopolysaccharide-induced acute kidney injury in the rat. BMC Nephrol 2012; 13: 25, doi: 10.1186/1471-2369-13-25.

23. Ziraldo C, Vodovotz $Y$, Namas RA, Almahmoud K, Tapias V, $\mathrm{Mi} Q$, et al. Central role for MCP-1/CCL2 in injury-induced inflammation revealed by in vitro, in silico, and clinical studies. PLoS One 2013; 8: e79804, doi: 10.1371/journal. pone.0079804.

24. Slimani $H$, Zhai $Y$, Yousif NG, Ao L, Zeng $Q$, Fullerton DA, et al. Enhanced monocyte chemoattractant protein-1 production in aging mice exaggerates cardiac depression during endotoxemia. Crit Care 2014; 18: 527, doi: 10.1186/s13054014-0527-8.

25. He J, Chen $Y$, Lin $Y$, Zhang W, Cai $Y$, Chen F, et al. Association study of MCP-1 promoter polymorphisms with the susceptibility and progression of sepsis. Plos One 2017; 12: e0176781, doi: 10.1371/journal.pone.0176781.

26. Kaplan J, Nowell M, Chima R, Zingarelli B. Pioglitazone reduces inflammation through inhibition of NF-kappaB in polymicrobial sepsis. Innate Immun 2014; 20: 519-528, doi: 10.1177/1753425913501565.

27. Bosmann M, Ward PA. The inflammatory response in sepsis. Trends Immunol 2013; 34: 129-136, doi: 10.1016/j.it.2012. 09.004 .

28. Suzuki M, Tsujikawa M, Itabe H, Du ZJ, Xie P, Matsumura N, et al. Chronic photo-oxidative stress and subsequent MCP-1 activation as causative factors for age-related macular degeneration. J Cell Sci 2012; 125: 2407-2415, doi: 10.1242/ jcs.097683.

29. Jung YJ, Lee AS, Nguyen-Thanh T, Kim D, Kang KP, Lee S, et al. SIRT2 regulates LPS-induced renal tubular CXCL2 and CCL2 expression. J Am Soc Nephrol 2015; 26: 15491560, doi: 10.1681/ASN.2014030226.

30. Wu S, Aksoy M, Shi J, Houbaviy HB. Evolution of the miR-290-295/miR-371-373 cluster family seed repertoire. PLoS One 2014; 9: e108519, doi: 10.1371/journal.pone. 0108519.

31. Zheng GX, Ravi A, Calabrese JM, Medeiros LA, Kirak O, Dennis LM, et al. A latent pro-survival function for the mir290-295 cluster in mouse embryonic stem cells. PLoS Genet 2011; 7: e1002054, doi: 10.1371/journal.pgen.1002054.

32. Medeiros LA, Dennis LM, Gill ME, Houbaviy H, Markoulaki $\mathrm{S}, \mathrm{Fu}$ D, et al. Mir-290-295 deficiency in mice results in partially penetrant embryonic lethality and germ cell defects. Proc Natl Acad Sci USA 2011; 108: 14163-14168, doi: 10.1073/pnas.1111241108.

33. Wang Y, Baskerville S, Shenoy A, Babiarz JE, Baehner L, Blelloch R. Embryonic stem cell-specific microRNAs regulate the G1-S transition and promote rapid proliferation. Nat Genet 2008; 40: 1478-1483, doi: 10.1038/ng.250.

34. Ma H, Wang $X$, Ha T, Gao M, Liu L, Wang $R$, et al. MicroRNA-125b prevents cardiac dysfunction in polymicrobial sepsis by targeting TRAF6-mediated nuclear factor kappaB Activation and p53-mediated apoptotic signaling. $J$ Infect Dis 2016; 214: 1773-1783, doi: 10.1093/infdis/jiw449. 